\title{
The Remote Monitoring System Design of Farmland Based on ZigBee and GPRS
}

\author{
Jinhu Liao, Qingyong Zhang, Yang Fang, Xuegang Xu, \\ School of Automation, Wuhan University of Technology Wuhan, 430070 China \\ liaojinhu@whut.edu.cn
}

Keywords: ZigBee; GPRS; Intelligent Agriculture; Remote Monitoring

\begin{abstract}
A remote monitoring system is designed, which can remote monitor farmland in real time and make informed management decisions. The system collects data of farmland by using ZigBee modules, makes data fused by using high performance controller ARM microcontroller, transfers data to remote computer by using GPRS modules, and makes informed management decisions by computer. Not only can the solution improve the level of agricultural production, but it can also reduce human costs effectively.
\end{abstract}

\section{Introduction}

Agriculture has been the basis of material production in our country, and China also has been an agriculture country [1-5]. Collecting the field information in real time, fast and continuously is an important basis of intelligent agriculture, but considering the limitations of cable monitoring system and the features of agriculture including regional decentralization, various objects, laggard communication uncertain environmental factors and evidently effected by environment, we can't monitor crops' growing environment timely and effectively, such as illumination, CO2 concentrations and temperature and humidity of soil [6-10]. It will cause crops getting unreasonable irrigation and supplement fertilizer, which will reduce the output of crops and even kill the crops. Considering the condition of monitoring field, we use ZigBee modules, GPRS modules and computer controlling comprehensive, propose a remote monitoring scheme, which can monitor and manage the parameter of field, and meet the needs of information management in remote region.

\section{ZigBee}

ZigBee is the most widely used in many wireless sensor network technology currently. ZigBee is essentially a protocol, which is based on 802.15.4 wireless standard approved by IEEE and relates to wireless networks, wireless security and wireless application. It is mainly suitable for transferring small data, can embed various devices and have location-aware browsing, working at $2.4 \mathrm{GHz}$, $868 \mathrm{MHz}$ and $915 \mathrm{MHz}$ frequency bands, with the highest $250 \mathrm{kbit} / \mathrm{s}, 20 \mathrm{kbit} / \mathrm{s}$ and $40 \mathrm{kbit} / \mathrm{s}$ transmission rate. Comparing with other transmission technology, the features here is unique to it.

(1) Low-power

ZigBee can work at sleep mode. In sleep mode, ZigBee can save electricity and work for 6 months only powered by two batteries.

(2) Large capacity of the network

A ZigBee network can contain $255 \mathrm{ZigBee,} \mathrm{one} \mathrm{of} \mathrm{which} \mathrm{is} \mathrm{the} \mathrm{coordinator,} \mathrm{the} \mathrm{rests} \mathrm{are} \mathrm{router}$ or endpoint. If it builds a network through network coordinator, the network can contain 64000 ZigBee devices.

\section{System Design}

The framework of the remote monitoring and management system for farmland is shown in Fig. 1. The system is composed of two parts including wireless monitoring network and remote control center. In wireless monitoring network, there are many ZigBee endpoints and ZigBee coordinators 
existing in farmland. The ZigBee endpoints are in charge of collecting illumination, $\mathrm{CO} 2$ concentrations and temperature-humidity of soil, transferring data to ZigBee coordinators through ZigBee wireless network. The ZigBee coordinators will forward data to GPRS gateway, which will fuse data in order to decrease data sizes and send data to remote control center. In remote control center, host computer will receive and resolve data, then display the monitored data.

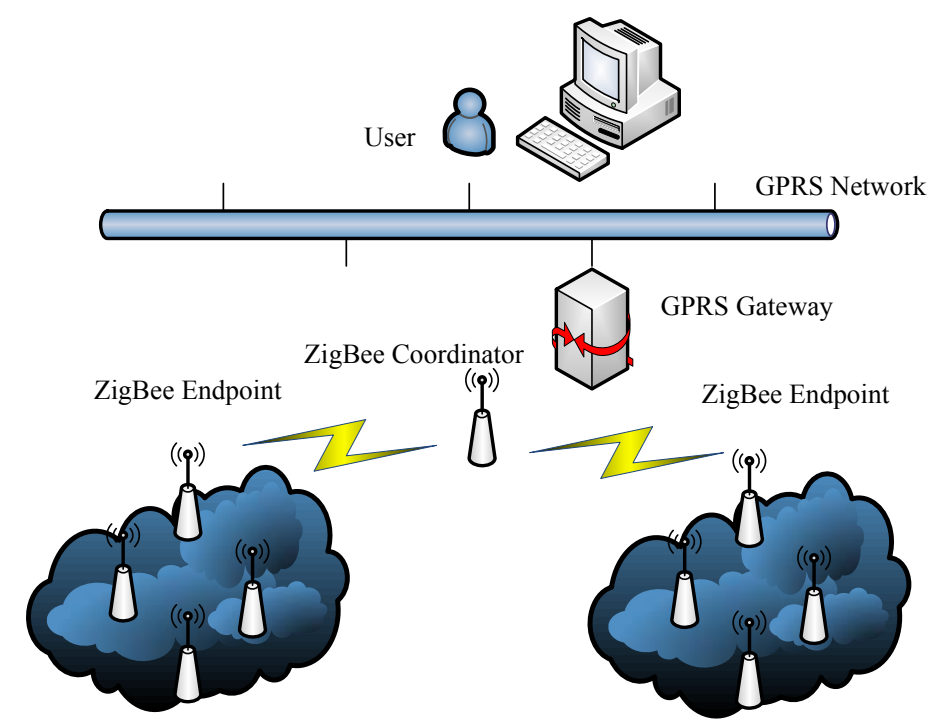

Fig. 1 System Diagram

\section{The Main Module}

A. ZigBee Module

a. Hardware Selection

There are two kinds of solutions about ZigBee hardware on the market at present, one of solutions is ZigBee and MCU double-chips solution and the other is single-chip. For double-chips solution, it has advantage on saving energy, but we must develop protocol stack from underlying code which is a heavy work. For single-chip solutions, there are many existing products we can choose. In order to save costs and simplify system, we choose the SOC single-chip solution produced by TI, whose controller is wireless RF chip CC2530. The CC2530 contains industry-leading RF transceiver and enhanced 8051 core, supports ISP function, has consumption extremely low, and its transmission speed reach $250 \mathrm{kbit} / \mathrm{s}$. The software uses an open protocol stack ZStack produced by TI, whose opening decreases costs, speeds up the development and improve the flexibility of network.

\section{b. ZigBee Endpoint Design}

In ZigBee endpoint, CC2530 is chosen to be a controller, which runs OSAL operating system, collects data from sensors and transfers these data to ZigBee coordinators by ZigBee protocol. About sensors, we choose the low-power CO2 sensor S-100H, high resolution optical sensor TSL230B and high precision temperature and humidity sensor SHT75, and the controller communicate with these sensors by using simulated communication protocol.

\section{B. GPRS Gateway}

The framework of GPRS Gateway is shown in Fig. 2. Comparing with ZigBee endpoints, the GPRS gateway need strong data processing ability and running speed, so TI company's 32-bit TM4C123BH6PGEI microcontroller is chosen to be a core controller. It is an ARM CortexM4 core high performance microcontroller with $1.08-1.32$ chip voltage, $80 \mathrm{MHz}$ main frequency, $256 \mathrm{~KB}$ Flash and 32K SRAM. This core controller communicates with ZigBee coordinator, receives data from it, fuses data in order to decrease data sizes and controls GPRS module by using AT command. The GPRS will contact to GPRS network through PPP protocol and get IP address distributed by network operator, which will help GPRS module communicate with remote control center. After 
GPRS contacts to center, the GPRS module will transfer processed data to center with TCP/IP. During setting GPRS, we need input computer's IP address by using matrix keyboard and LCD will display these contents.

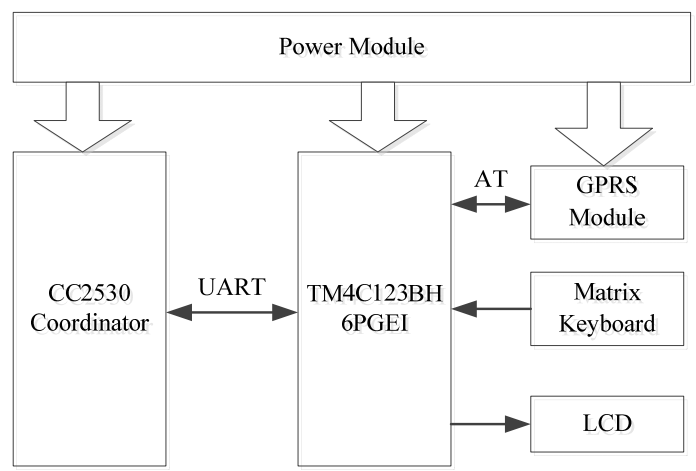

Fig. 2 GPRS Gateway’s System Diagram

\section{Software Design}

We use TI company's IAR Embedded workbench to develop each module's software and debug software by using JATG which is integrated in chips. In order to build a safe and stable ZigBee network, we need design ZigBee endpoint and coordinator's software based on Zstack. At the same time, we need to finish the design of GPRS gateway by using TM4C123BH6PGEI and GPRS module

A. ZigBee endpoint software design

ZigBee endpoint's software flow chart is shown in Fig. 3(a). The ZigBee endpoint is in charge of collecting sensors' data. During the initialization stage, the system initializes not only MCU peripheral modules but also OSAL operating system and adds tasks during initializing OSAL. During the collection stage, CC2530 will work for sleep mode after finishing data transmission and wait for being triggered by trigger signal, which is receiving sensors' data again.

B. ZigBee coordinator software design

ZigBee coordinator's software flow chart is shown in Fig. 3(b). The ZigBee coordinator is in charge of building the ZigBee network and transmitting data. During the data transmission, CC2530 will work for sleep mode when not receiving data and wait for being triggered by trigger signal, which is receiving ZigBee endpoints' data.

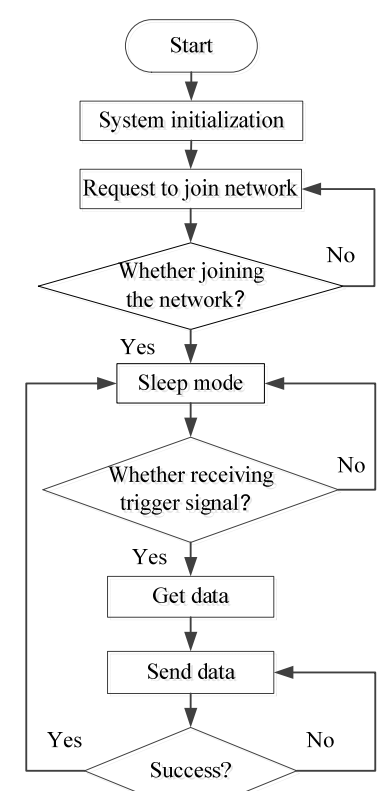

(a) ZigBee Endpoint Software's Flow Chart

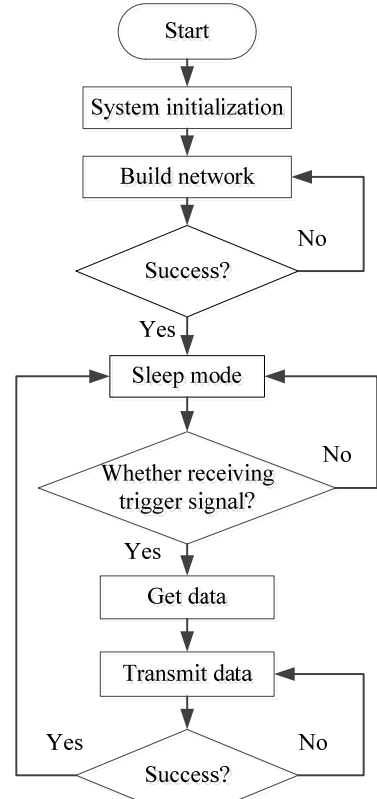

(b) ZigBee Coordinator Software's Flow Chart Fig. 3 ZigBee Software's Flow Chart 


\section{GPRS gateway software design}

GPRS gateway' software flow chart is shown in Fig. 4. The GPRS gateway is in charge of receiving ZigBee coordinator' data, fusing these data and sending the data to remote control center with TCP/IP protocol. During the initialization stage, we initialize GPRS module by using AT instruction and fuse data by using data compression algorithm.

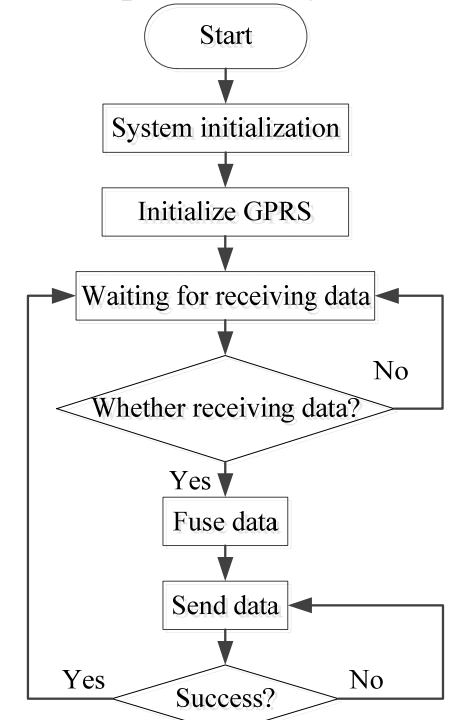

Fig. 4 GPRS Gateway Software's Flow Chart

\section{Summary}

Through using ZigBee and GPRS technology, the system finishes data collection and transmission. Comparing with traditional monitoring scheme, ZigBee and GPRS solution has strong scalability and flexibility especially in a harsh environment, and it can improve the monitoring mode effectively and decrease human cost to some extent.

\section{Acknowledgments}

The paper is supported by Independent Innovation Foundation Programs of WHUT.

Author to whom correspondence should be addressed. Electronic mail: qyzhang@whut.edu.cn

\section{References}

[1] Jiang Liming, Zhuang Weidong, "Study on the ZigBee/GPRS Technology in Precision Agriculture," Journal of Agricultural Mechanization Research, 2013, 4:179-182.

[2] Liu Qing, Gong Qiang, "Design of Intelligent Wireless Sensor Networks for Greenhouses with ZigBee Based on CC2530,” Journal of Mianyang Normal University, 2015, 2(34):25-29.

[3] Yang Yinghong, Wang Lichun, Mao Zengchuang, "Research on the Control System of Intelligent Greenhouse Based on ZigBee," Electronic Test, 2015, 17:14-17.

[4] Li Xianglin, Hu Mei, Li Ying, "Intelligent Water Saving Irrigation System Based On ZigBee," Journal of Lanzhou Polytechnic College, 2011, 3(18):11-17.

[5] Jiang Yuqi, Gu Zhaogui, Shi Wangwang, "Multifunctional Wireless Measurement and Control System Based on ZigBee and GPRS," Journal of Mechanical \& Electrical Engineering, 2014, 6(31):814-818.

[6] Zhang huiying, "Design of Intelligent Monitoring System in Greenhouse Based on IOT," Hubei Agricultural Sciences, 2014, 14(53):3402-3406, 3411.

[7] Lin Yuanguai, Long Shunyu, Yang wei, "Intelligent Agriculture Application System Based on 
IOT Technoligy," Internet of Things Technologies, 2013, 3:71-74.

[8] He Yong, Nie Pengcheng, Liu Fei, "Advancement and Trend of Internet of Things in Agriculture and Sensing Instrument," Transactions of the Chinese Society for Agricultural Machinery, 2013, 10(44):216-226.

[9] Sun Bing, "Remote Agricultural Environment Monitoring System Design," Electronic Test, 2015, 15:21-23.

[10] Hu Mei, Wang Yongxi, Liu Baolu, "Automatic Water-saving Irrigation Intelligent System Based on ZigBee," Automation \& Instrumentation, 2014, 2:116-117. 